\title{
SPACEBAR
}

\section{KINEMATIC DESIGN BY COMPUTER GRAPHICS}

R1chard J. R1cc1

Automation Development and Loft Group

Lockheed-California Company

Burbank, California

\section{SUMMARY}

The development of kinematic mechanisms on the design board is often an extremely complex and time-consuming task. It was this impetus which led to the development of the interactive graphics program SPACEBAR at Lockheed.

The SPACEBAR program allows the direct design and analysis of mechanisms right at the terminal screen. All variables including linkage geometry, st1ffness, and applied loading conditions can be input and/or changed from the terminal. All data can be described and displayed in three dimensions. All mechanism configurations can be cycled through their range of travel and viewed in their various geometric positions, again in three dimensions. Output data Includes geometric positioning in orthogonal coordinates of each node point In the mechanism, velocity and acceleration of the mechanism node points throughout 1ts range of travel, and internal loads and displacements of the Individual node points and linkages. All analysis calculations are performed at the scope and take, at most, a few seconds to complete. Output data can be viewed at the scope and also printed at the discretion of the user.

\section{INTRODUCTION}

The designing of light weight kinematic mechanisms such as control systems, landing gears, and door hinges is a major area of development in the design of aircraft and their subsystems.

Developing these complex mechanisms and control systems and predicting their motion is and always has been one of the more challenging tasks for the design engineer. Once beyond two-dimensional kinematic motion, geometric design techniques quickly increase in complexity and the chances for system inaccuracies multiply. The board work required for even relatively simple designs becomes a maze of overlays upon overlays, and development time can turn Into weeks and even months. Often, complicated analytical calculations to determine loads and particularly deflections, are nearly nonexistent until the final design stage has been attained. As a result, it is usually too late in a schedule to make the changes desired to optimize the weight-load relationship. 
A new method was needed to overcome this labyrinth of drawings and calculations and to speed up the iterative procedure for arriving at a final design. A way in which periodic checks of loads and deflections could be made without consuming days of development time was the basis that led to the development of SPACEBAR in computer graphics at Lockheed.

\section{Computer Graphics Development}

Lockheed began investigating the applications of computer graphics to design and manufacturing in the 1960 's. It was soon apparent that computer graphics could reduce development time and cost, and alleviate many of the engineer's tedious everyday tasks. In the business world, this had been the main thmist of computer development, but in engineering, the emphasis had been concentrated on solving the bigger and more complex analytical problems. The everyday task was still a millstone around the engineer's neck. With the advent of interactive CRT's and the proper software, however, Lockheed felt that many of these chores could now be automated.

\section{The Development of SPACEBAR}

Mechanism development was one of these chores; a design task which was fairly simple conceptually, but which consumed a great deal of time and effort in developing a solution. The program developed to help alleviate this problem was called SPACEBAR.

SPACEBAR is a three-dimensional kinematics design and analysis program developed specifically for computer graphics at the Lockheed-California Company. It was created to help accelerate and improve the design of mechanical systems, thereby reducing development cost and achieving a more optimal product. Although it was developed with an emphasis on aircraft systems, its capabilities allow the design of a large varlety of mechanisms including such items as control systems, landing gears, door hinge mechanisms, and packaging equipment. In fact, nearly any mechanical system which includes the use of IInkages, disk cams and tracks can be designed, at least in part, on SPACEBAR.

SPACEBAR is an interactive computer graphics engineering program. It enables the user to vary any system parameter at will, and to evaluate the newly developed configuration, as displayed on the terminal screen, in a matter of seconds. The program allows the modification of all mechanism geometric parameters such as link lengths, fixed axle locations, rider point locations, and cam and track curve descriptions. This manipulated data is then used to generate a new geometric model. The program also allows the modification of all analytical stiffness and strength data associated with the physical mechanism. From this design data the program calculates and displays all the geometric and analytical information necessary for interactively designing kinematic systems. It determines system motion, internal member loads and system deflections, and displays this data in the physical relationship of the mechanism in three dimensions. 


\section{HARDWARE}

To operate the SPACEBAR program Lockheed uses e1ther an IBM 2250 or a Vector General graphics terminal on-1ine with an IBM 360-9I mainframe computer. A Data General Nova 800 is used to provide compatibility between the Vector General terminal and the IBM 360-91 mainframe. The mainframe computer is used for multiple tasks and the program operates in a $130 \mathrm{k}$ dedicated core space with a very high priority.

The graphics terminal provides three modes of communication between the user and the program (see Figure 1). The first of these is through the Function Key Box. The program can test which of 32 independent buttons contained in the function key box has been pressed and from this determine a course of action. This method is often used to bring up new subroutines contained within a program. In SPACEBAR the function key box is used solely for rotating the three-dimensional stick representation of the model being investigated. By pressing one of six active function key buttons, the user indicates to the program the desired direction of model rotation. Two buttons are used for rotation ( + or - ) about each of the system orthogonal axes. The second mode of communication with the program is the typewriter keyboard. Through this medium the user can enter any alphanumerical information desired. SPACEBAR uses the typewriter keyboard for entering mechanism geometric data, applied loads, and stiffnesses. The third method of communication is with a fiberoptic light-sensitive pen. In SPACEBAR the light pen is used to select data points to be changed or one of a "menu" of options to be activated. The options contain the many different analytical and geometrical subroutines which the program uses to evaluate the mechanism model.

Interactive response whlle operating SPACEBAR is very rapid; usually less than one or two tenths of a second. The only exception to this is during the generation of the inverse matrix used to determine system loads and deflections. This calculation can sometimes take up to several seconds for completion depending upon the mechanism being investigated.

The SPACEBAR program itself is one of several analysis programs contained in specified load modules. These load modules are stored on disk packs which can be routinely accessed from the terminal.

\section{HARDCOPY OUIPUT}

Both microfilm plots and standard printout can be generated from the SPACEBAR program. In the analysis phase of the program, printout is generated automatically. In the geometric phase of the program, using the light pen to detect a screen menu labeled "PRINT" will generate the desired printout. For microfilm plots the user must first inform the computer operator that plots are to be inftiated. The operator then loads a plot tape on a tape drive and this tape drive is dedicated to the SPACEBAR program during its operation. The user is then free to select that information for which plots are desired. By using the light pen to detect a screen menu labeled "PLOT", microfilm plot data, duplicating the picture shown on the terminal screen, is output onto 
the dedicated plot tape. Upon completion of the session's work, the data on the plot tape is converted into microfilm from which hardcopy can be generated using a Xerox process.

\section{CAPABIIITIES}

The analysis capabilities of the SPACEBAR program are extensive and provision is made to query virtually any parameter of the mechanism the user may wish to study. The types of parameters to be studied can be broken down into two basic sets: geometric or motion parameters, and analytic or structural parameters.

\section{CAPABILITIES - GEOMETRIC}

The SPACEBAR program has the geometric capability of integrating into a unified three-dimensional mechanical system such diverse mechanical devices as the 4-bar linkage, elther by 1tself or with a rider point, the cam with cam follower, and the track. It can then display the operational characteristics of this integrated system on the terminal screen. Finally, it can store this mechanical system for later retrieval and study.

\section{Model Description and Restrictions}

The basic units for any mechanism used in the SPACEBAR program are the Fourbar linkages (Figure 2). All other mechanism devices are "attached" or interlinked with these. In any single case the program can handle up to four interlinked fourbar linkages. Rider points can be attached to any or all of these.

A given fourbar linkage may be "tied" to the rider point of the previous fourbar by use of a "tie" switch. This will tie the motion of the following fourbar to the previous linkage as follows: the rider point of the previous linkage is analogous to end point of the first bar of the following linkage (see Figure 3). In effect this results in a simulated variable length first bar in the second fourbar linkage.

Disk cams and cam followers are interlinked to fourbar linkages by use of the "cam insert" feature (Figure 4). The only constraint on cams is that they cannot be free to move in space but must rotate about a fixed axle point. All cams and cam followers are described by a series of points to which a spline curve is then fit. The spline fit curve is based on a piecewise cubic with continuous first and second derivatives. Each mechanism in SPACEBAR can have up to four cams. In the analysis portion of the program each cam-camfollower arrangement is analyzed as an instantaneous fourbar at a given angular position.

Tracks in SPACEBAR are defined by the motion of a link end point on a fixed track centerline. The track centerline can be either straight or curved in three-dimensional space and is represented by the intersection of two surfaces (e.g., the intersection of two planes results in a straight line). 
Fourbar linkages are "tied" to tracks in the same manner as they are tied to rider points, except in this instance it is the end of the second link in the preceding fourbar which is tied to the track (see Figure 5).

\section{Data Input}

Data required to establish a mechanical system in SPACEBAR can be entered in two ways. The first is by submitting a punched card deck to a batch program. The batch program then enters the mechanism's parameters onto a data set accessible by the program. For any new system with an extensive and complicated linkage, the submitting of axle location, link lengths, and stiffness data by punched cards will save a considerable amount of terminal usage time. A manual has been developed for the user which describes the method and order of data submittal.

The second method of entering data is through the terminal itself. Selection of the menu "INPUT" (Figure 6) will bring up the input data display for the mechanism under investigation (Figure 7). Any data point in this menu can be changed by simply light pen selecting that value and keying in a new value on the typewriter keyboard. This method of entering data is particularly useful for refinement of the mechanism and the resulting case can be used instantly at the terminal or saved on a disk pack for future reference.

\section{Display Modes}

Two display modes are available in SPACEBAR to help the user attain a better understanding of the mechanism that is being created. The first is a display of the mechanical system showing both the plan and side views

(Figure 6). Included in this display are the X, Y, and $Z$ coordinates of each point in the model, the angle through which each axle in the mechanism rotates with respect to its reference position, the initial and final input and output angles through which the mechanism is desired to traverse, and the delta angle through which the mechanism will step when cycled. Also included in this first display is a graph depicting the desired (and after the mechanism has been cycled, the actual) input-output angular motion relationship.

The second display mode depicts the mechanism in a three-dimensional axenometric projection (Figure 8). This three-dimensional stick model representation may be rotated about any of three orthogonal axes until the desired projection is achleved. The rotation can be accomplished either by using the function key box or by keying in the desired angles on the typewriter keyboard. A magnification option exists which allows the user to blow up any desired portion of the mechanism for a more detalled examination of 1ts operat1on. In both of these displays the mechanism can be cycled or stepped through its desired range of motion with the pletorial presentation of the mechanism changing accordingly. If the mechanism is unable to traverse the given range of motion, the system will stop at the last attainable position and a note will appear indicating an impossible geometric relationship has been asked for. 


\section{Geometric Data Output}

The methods developed to enter and change data in the SPACEBAR program have proven extremely useful to the design and analysis engineers. It allows them the freedom to check and modify the geometric characteristics of any parameter of the system at will and in a few minutes of time.

A user can cycle the particular system, check out his geometric and analytic characteristics in seconds and determine any deficiencies. Then, if deficiencies or improvements are determined, corrections can be made right at the terminal, again in seconds. The new system model is immediately available to be analyzed. The whole process from system modification to geometric and analytical checkout takes but a few minutes of terminal time.

The type of geometrlc data output on SPACEBAR includes all system nodal point locations, nodal point velocities, and nodal point accelerations. This data is available to the user at any geometric positioning of the mechanism within its range of travel. There are two ways in which this output data is displayed. The first is located within the plan and side view mechanism geometry display discussed above under display modes (Figure 6). It includes the $\mathrm{X}, \mathrm{Y}$, and $\mathrm{Z}$ coordinates of each nodel point in the system. These values change accordingly as the mechanism is cycled or stepped through its range of travel and always records the current geometric position of each nodal point during the cycling sequence. Along with the $X, Y, Z$ coordinate display is a graph depleting the relative motion of the mechanism input angle vs. Its output angle. An enlarged view of this graph is available under a memu option labeled "GRAPH" which the user may select. The input-output graph can contain up to three separate curves depending on the user's needs. The first curve is empirical data that is input by the user and represents the desired relationship between the input and output angular motion of the system under development. This data can be input by punched cards or at the terminal. The second curve is calculated internally by the program as the mechanism is cycled and represents the actual relationship of the input vs. output angle. The third curve is generated when the mechanism is cycled again. This is usually done after the mechanism has been modifled and thereby allows the user to compare the motion of the last two developed mechanisms with the desired input curve. Each time the system is cycled the oldest of the two internally generated curves is erased and a new curve based on the motion of the currently displayed mechanism is generated.

The second method of displaying geometric output data is contained in a display labeled "VARIABLE SELECTOR" which is similar to the geometry display. This display is used to compare the relationship between varlous geometric parameters while the mechanism is cycled through its range of travel. The relationship is presented in the form of a graph at the top half of the terminal screen. The variables to be compared are selected by the user through the use of the light pen and Include the X, Y, or $Z$ of the angular positioning, velocity, or acceleration of any nodal point in the mechanism. The user may change these parameters at will and obtain a new graph. 
Data entered either by punched cards or at the terminal can be stored in the SPACEBAR program. Two separate files exist for this purpose. Only card Input is stored on the first file. The user accesses this file by light pen selecting the option "NEW CASE" avallable on most of the SPACEBAR displays. Changes can be made to a case from this file and the modified case may be saved in the second file. The second file is accessed by light pen selecting the word "FILE". The user may delete, modify, or add cases to this second 11le. There also is a hidden code name which may be added to cases in the second file to prevent unwanted modifications. Each of these two f1les can store up to 100 different cases (200 cases total).

\section{CAPABILITIES - ANALYTIC}

Once the geometric parameters of a mechanism have been established, the user proceeds to the analysis portion of the program where an analytical or structural evaluation of the system can be made. The analysis portion is divided into two parts. First is the determination of linkage internal loads and system support point reactions from externally applied loads; second is the calculation of system deflections due to externally applied loads. Two types of external load conditions can be applied to a given mechanism in SPACEBAR: Jam loads and applied loads.

\section{Internal Loads - Jam Loads}

The jam load condition consists of fixing the mechanism from rotating at the last axle point and applying a $112.98 \mathrm{~m}-\mathrm{N}$ (1000 in-lb) moment at the first axle point. Since there is only one load path possible the mechanism is always determinent. The internal loads are then calculated by simple structural joint vector analysis. The entire process is accomplished by a geometric matrix which is automatically set up by the SPACEBAR program and which varies according to the size and component makeup of the mechanism. This geometric matrix is then multiplied by a special jam loads matrix to determine the system Internal loads.

The user has the option of asking for two sets of jammed loads answers. The first set of answers consists of the load in each link and at each support point, the moment at each intermediate axle, and the input-output mechanical advantage of the mechanism at the selected angular position. This data is displayed in tabular form under the "Internal Loads - Jammed" menu of the program (Figure 9). The second set of answers consists of a plot of the load in a given link or at a selected support point vs. the mechanism input angle over the range of travel requested. This graph is displayed under the menu "JAM LOAD SELECTOR" which is similar to the geometry display and allows the user to select the particular parameter to be investigated.

\section{Internal Loads - Applied Loads}

The applied loads conditions consist of fixing the mechanism at the first axle point and applying loads wherever desired throughout the mechanism Provision is made under the applied loads menu (Figure 10) to add or delete 
a load at any system node point by light pen selecting the node desired and keying in the load. Loads are applied along the basic X, Y, $\mathrm{Z}$ orthogonal axis of the mechanism. The internal loads for the applied loads condition are calculated similarly to that of the jam loads condition. A geometric matrix is constructed which resembles that of the jam loads geometric matrix and an applied loads matrix is multiplied by that geometric matrix to determine the internal loads. Again, this is all done internally by the program. Resultant internal loads are displayed on the "Internal Loads-Applied" menu of the program and resemble very closely that of the "Internal Ioads-Jammed" menu。

\section{System Deflections}

The deflections of a selected mechanism are generated similarly to that for the internal loads condition. In this circumstance, however, the system stiffness parameters as well as the geometric parameters and applied loads must be established. Linkage areas and inertias as well as support point spring rates must be supplied for the given mechanism. This data is entered either by punched cards or at the terminal under the two displays "AREA AND INERTIA" and "SPRING SUPPORTS" (FIgures 11 and 12). Data submitted by punched cards must be entered through the batch program, while data submitted at the terminal uses the light pen and typewriter keyboard. Once all required stiffness data has been entered into the program, the deflection calculations can be made. The user has the option of calculating two different sets of deflections. The first deflection set is based on a mechanism jammed at the last axle with a $112.98 \mathrm{~m}-\mathrm{N}$ (1000 in-lb) torque applied on the first axle. This will give the user the total stiffness of the mechanism being investigated as well as display the $\mathrm{X}, \mathrm{Y}$, and $\mathrm{Z}$ deflections of each individual node point (Figure 13). The second set of deflections will be based on any applied loads which were entered under the applied loads menu of the program. It also displays the deflection of each individual node point.

In all the calculations discussed above, the user may generate the prescribed data in any mechanism position he chooses. He merely sets the linkage position in the geometry section of the program and then asks for the data in the analytic section of the program.

\section{Internal Program Operation}

To calculate the analytical data described above, SPACEBAR sets up internally and automatically a series of finite element type flexibility and stiffness matrices. A stick model detalling the system and element members of a typical mechanism is shown in Figure 14. Once the basic flexibility matrix has been established, it is multiplied by the applied loads or jam loads matrix to determine the resultant system loads and deflections.

$$
[\mathrm{f}]=[\mathrm{E}]^{-1}[\mathrm{AL}]
$$




$$
\begin{aligned}
{[u]=[\mathrm{K}]^{-1}[\mathrm{AL}] } & \\
\text { where: } \mathrm{E} & =\text { geometry matrix } \\
\mathrm{K} & =\text { stiffness matrix } \\
\mathrm{AL} & =\text { applied loads matrix } \\
\mathrm{u} & =\text { nodal displacements } \\
\mathrm{f} & =\text { nodal internal loads }
\end{aligned}
$$

The largest matrix which an individual SPACEBAR case can generate is of the order of $60 \times 60$. This is equivalent to four interconnected fourbar linkages, each with an independent rider point. SPACEBAR also, however, has the capability of transferring output data from one case as input data into another case. In this way very large mechanisms can be handled piece by piece.

\section{Present Usage}

SPACEBAR's primary usage to date has been to check and verify the operating characteristics of several existing mechanical systems. These include simulation of the Navy S-3A horizontal stabilizer tab actuation mechanism, simulation of an airstairs for the L-1011 wide body Tristar airliner, and modification to the geometry of the S-3A landing gear door mechanism. In the last case, 1t was estimated that at least 10 hours of board design work was done in one and one-half hours of terminal time with the internal loads and deflection analyses as a bonus derived from using SPACEBAR. Correlation of the data from SPACEBAR with previous mamual methods was found to be very accurate and served to verlfy the program.

\section{FUTURE ENHANCEMENTS}

The SPACEBAR program, while a complete and useful tool in its present form, is still in its mechanical infancy. The addition during this last year of such features as cams, tracks, and a rider point to the basic fourbar linkage has greatly increased its versatility, and the inclusion of velocity and acceleration calculations along with some new data output displays has added to the system's data retrieval capabilities. However, there remains a multitude of major types of mechanical motions which are st1ll beyond the program's existing analysis techniques.

As SPACEBAR gains in usage and importance, more of these complex features $w 111$ be developed and incorporated. Mechanical devices simulating ldlers, floating links, and varlable length links controlled by spring stiffeners are all possible future enhancements. Actuators along with actuation motions and stiffnesses are also contemplated.

Other enhancements which look promising are a direct interface with CADAM (Computer Graphics Augmented Design and Manufacture), Lockheed's graphics drafting package, allowing the fast and efficlent transfer of mechanism geometrical parameters from one system to another and finally a direct hookup to a computer batch program where vibration and stabllity investigations can be carried out. 
All of these enhancements w1ll increase the versatility and capabllity of SPACEBAR with the eventual goal of designing and analyzing most, if not all, of the basic kinematic mechanisms by interactive computer.

\section{ACKNOWLEDGENENTS}

I wish to acknowledge the contributions of R1ck Fromm and Sy Southworth for the development of the original fourbar mechanism in SPACEBAR, and the contribution of Bridget Shycoff for her programing effort in the subsequent additions of the rider point, cam, and track mechanisms, and in the development of the velocity and acceleration subroutines. 


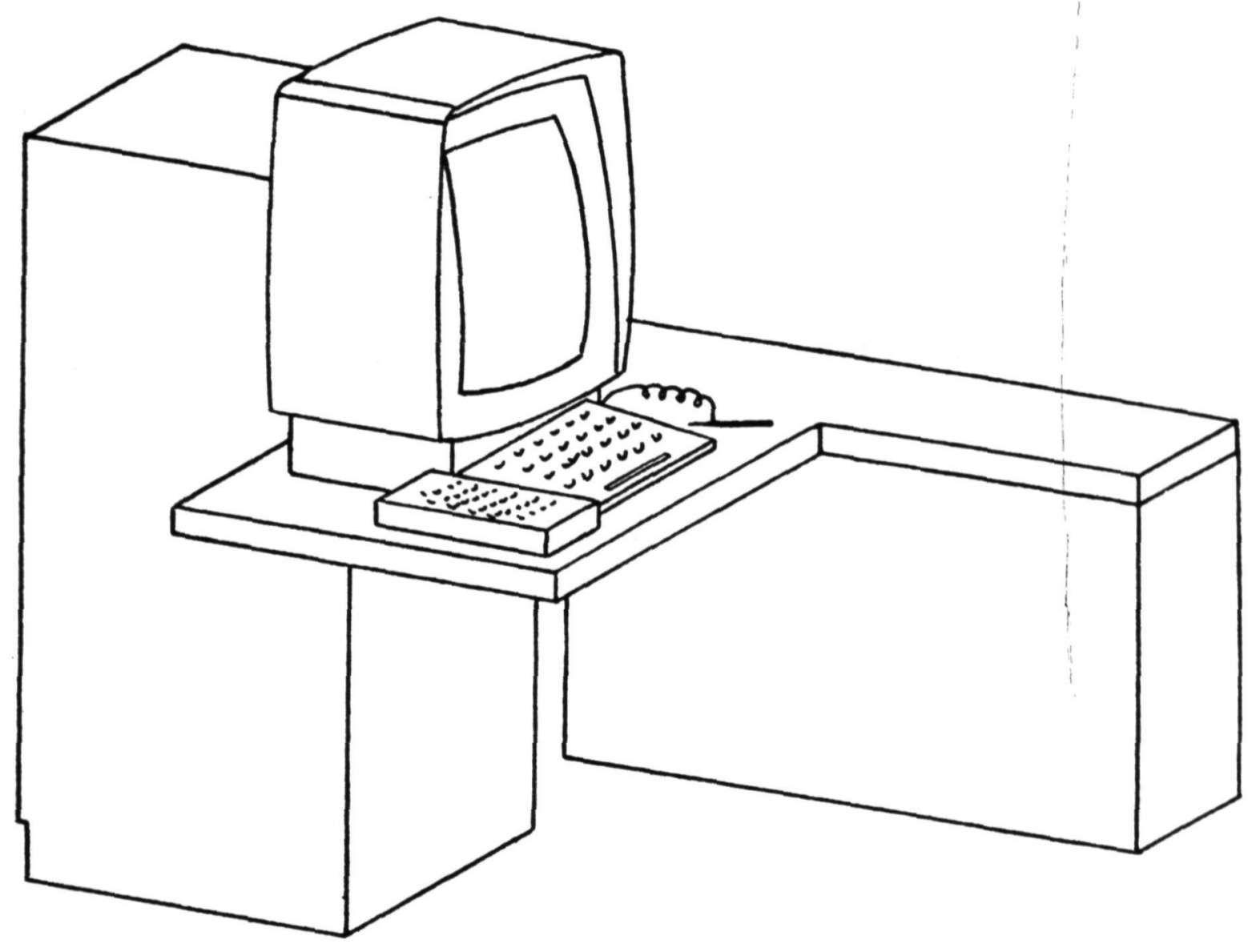

From left to right in the work area in front of the Cathode Ray Screen:

1. Function Key Console

2. Typewriter Console

3. Light Pen

Figure 1 - The IBM 2250 display unit. 


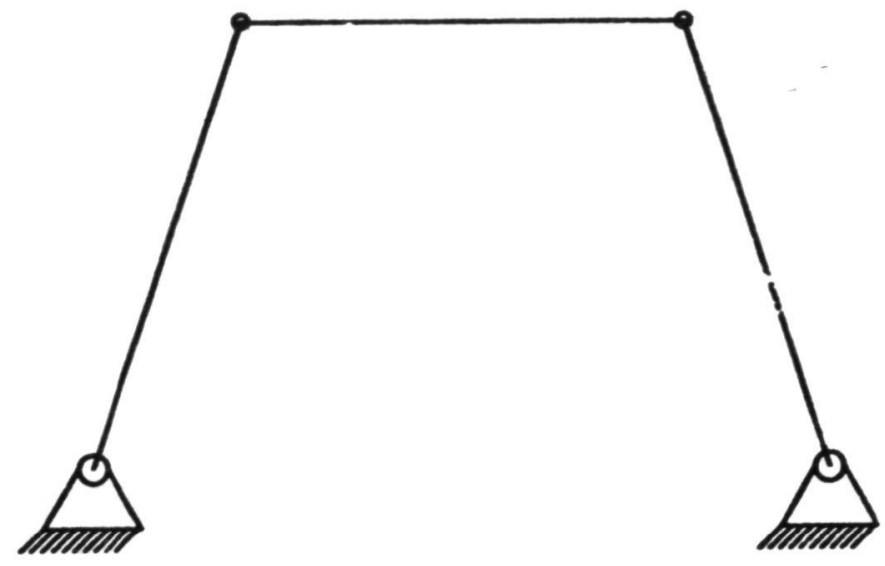

Figure 2 - Typical fourbar linkage.

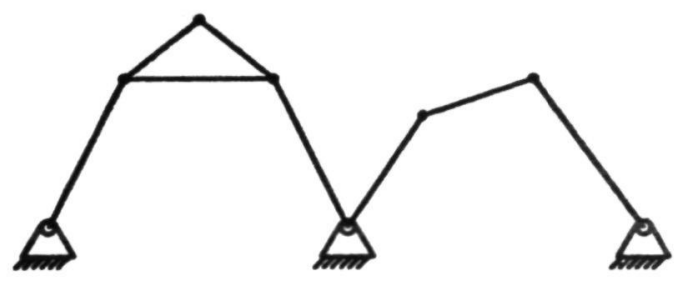

(A)

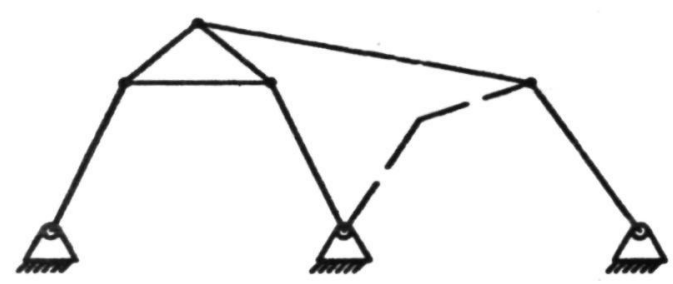

(B)

Figure 3 - "Tie" mechanism attaching following fourbar linkage to previous fourbar linkage rider point. 


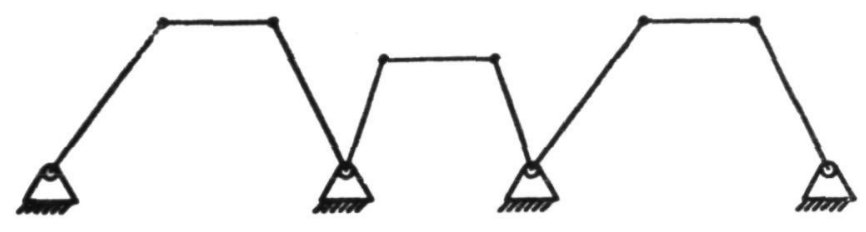

(A)

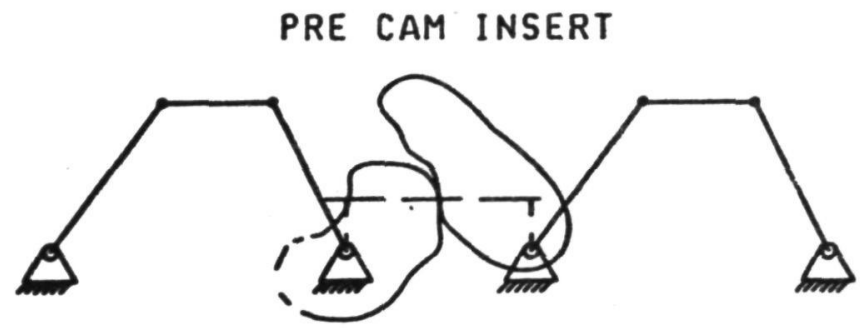

(B)

POST CAM INSERT

Figure 4 - "Tie" mechanism converting fourbar linkage into cam actuation device.

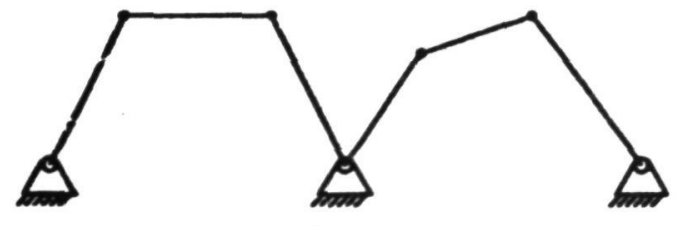

(A)

PRE TRACK TIE

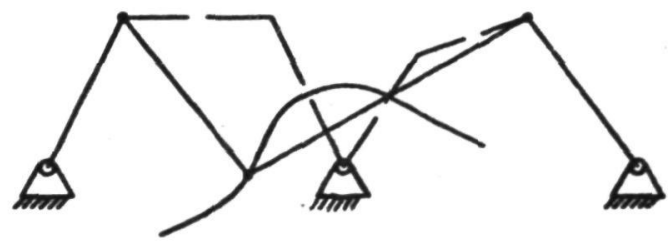

(B)

POST TRACK TIE

Figure 5 - Track "tie" mechanism attaching fourbar linkages to track centerline space curve. 


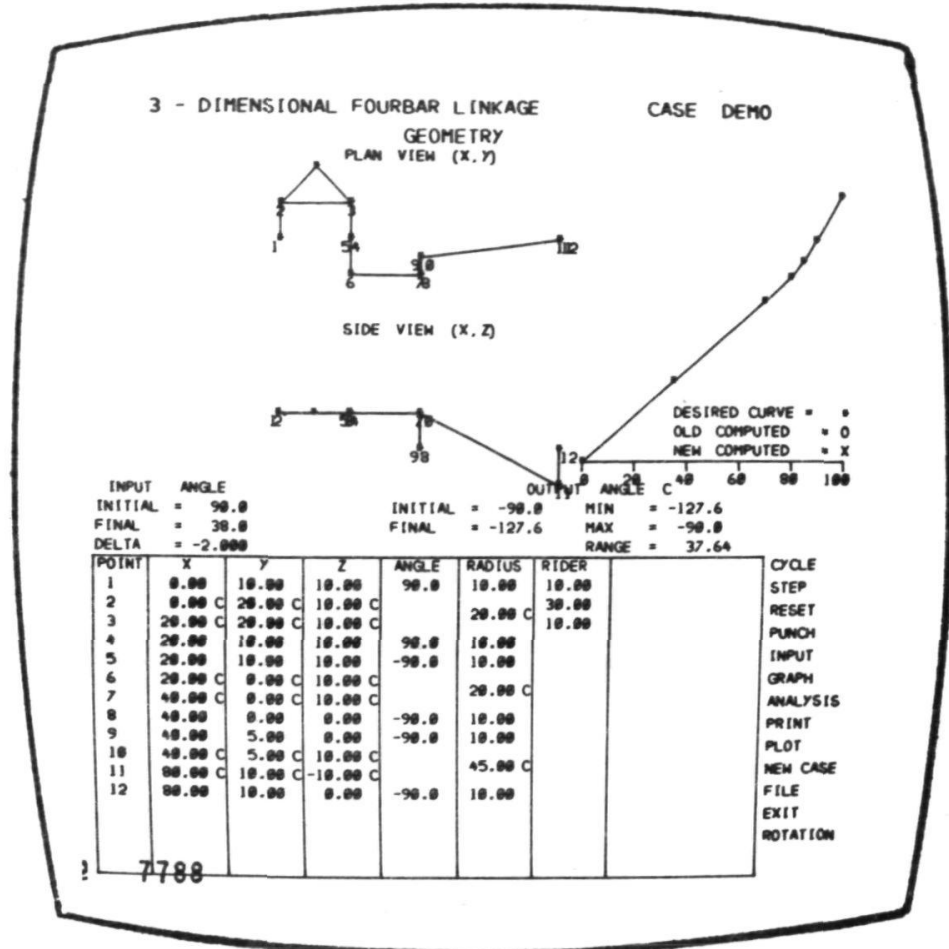

Flgure 6 - Geometry display - note list of menus along right hand side of screen. Light pen selection of these menus accesses various routines in the program.

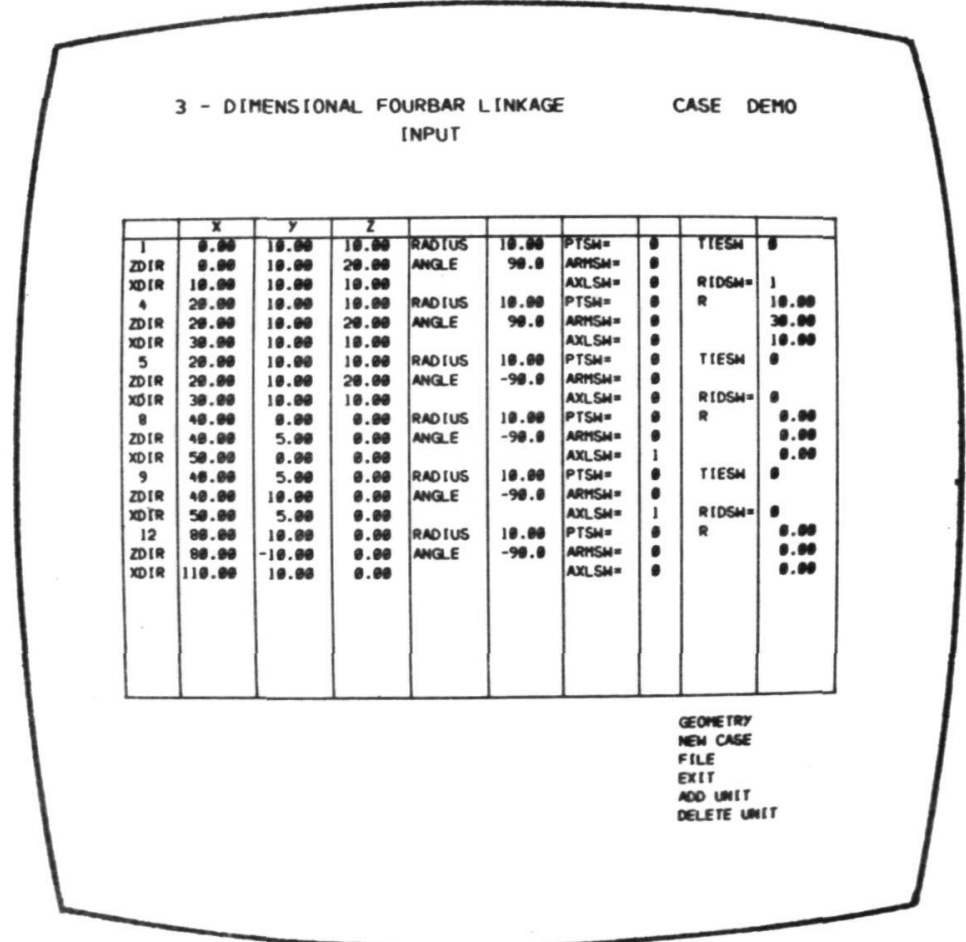

Figure 7 - Input display showing typical data description required for a given mechan1sm. 


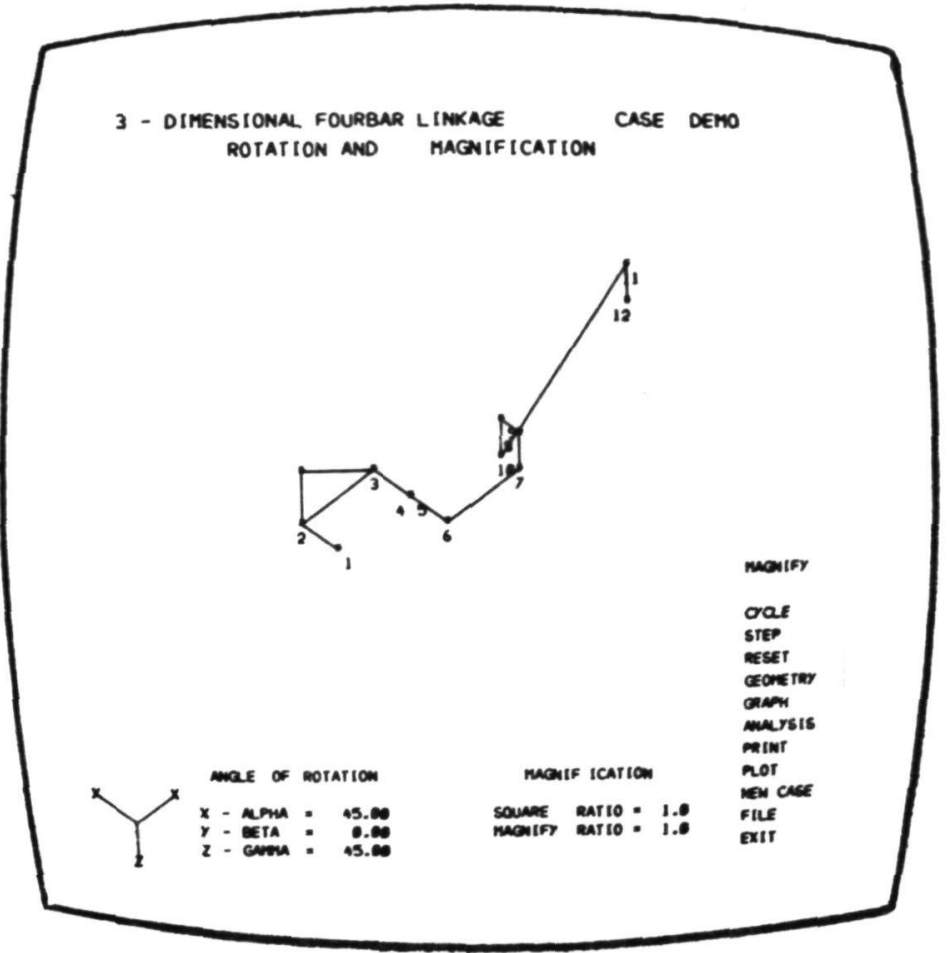

Figure 8 - Rotation display depicting axenometric view of given mechanism.

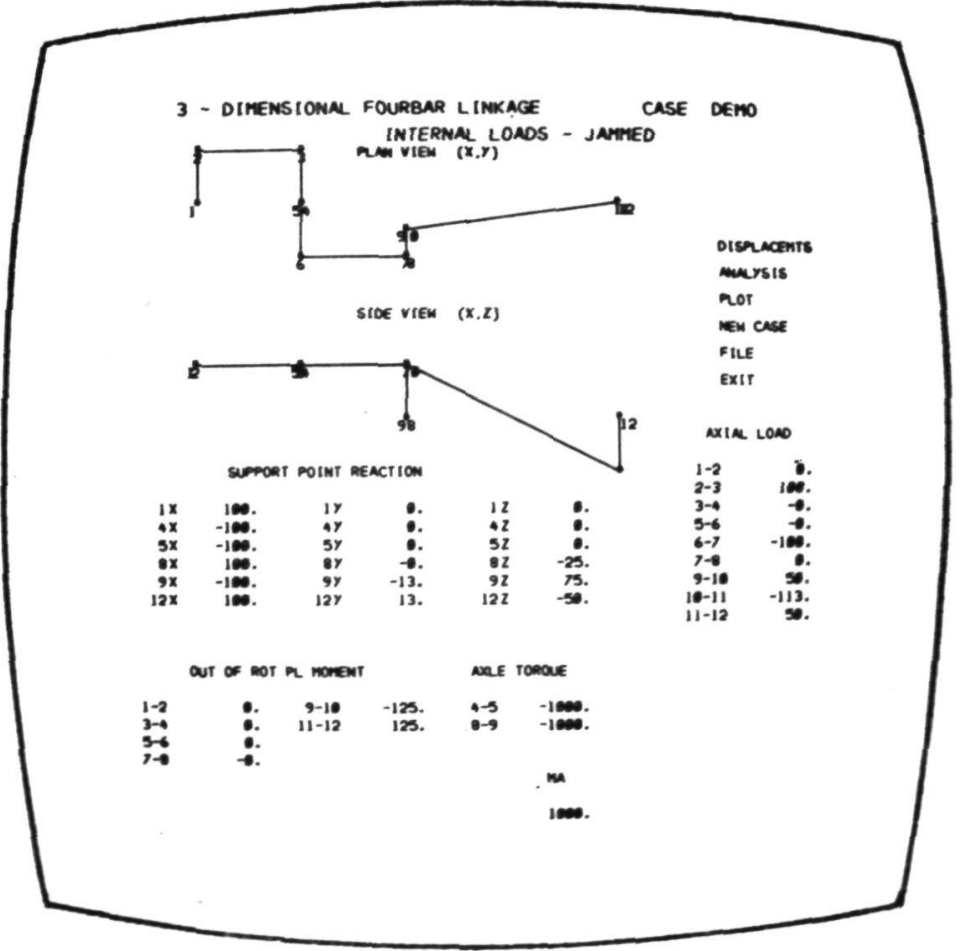

F1gure 9 - Internal loads - Jammed menu showing mechanism internal loads. 


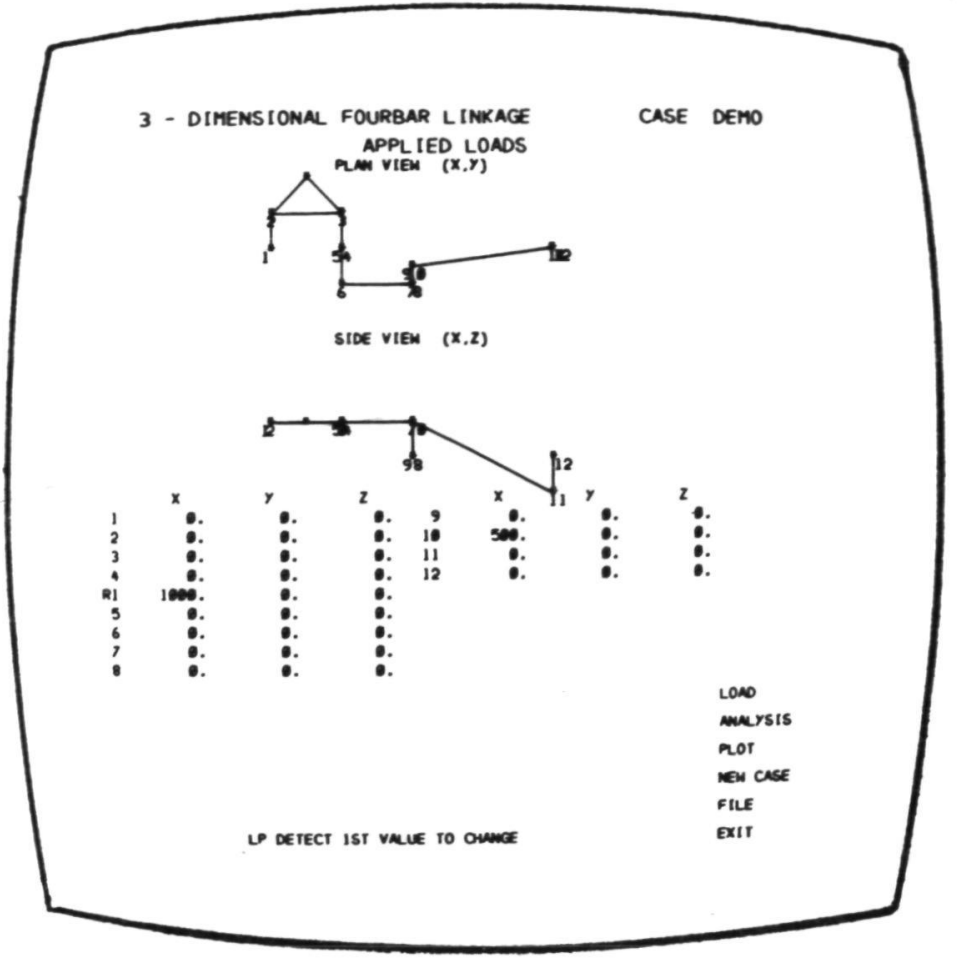

Figure 10 - Applied Loads menu showing mechanism and applied loads.

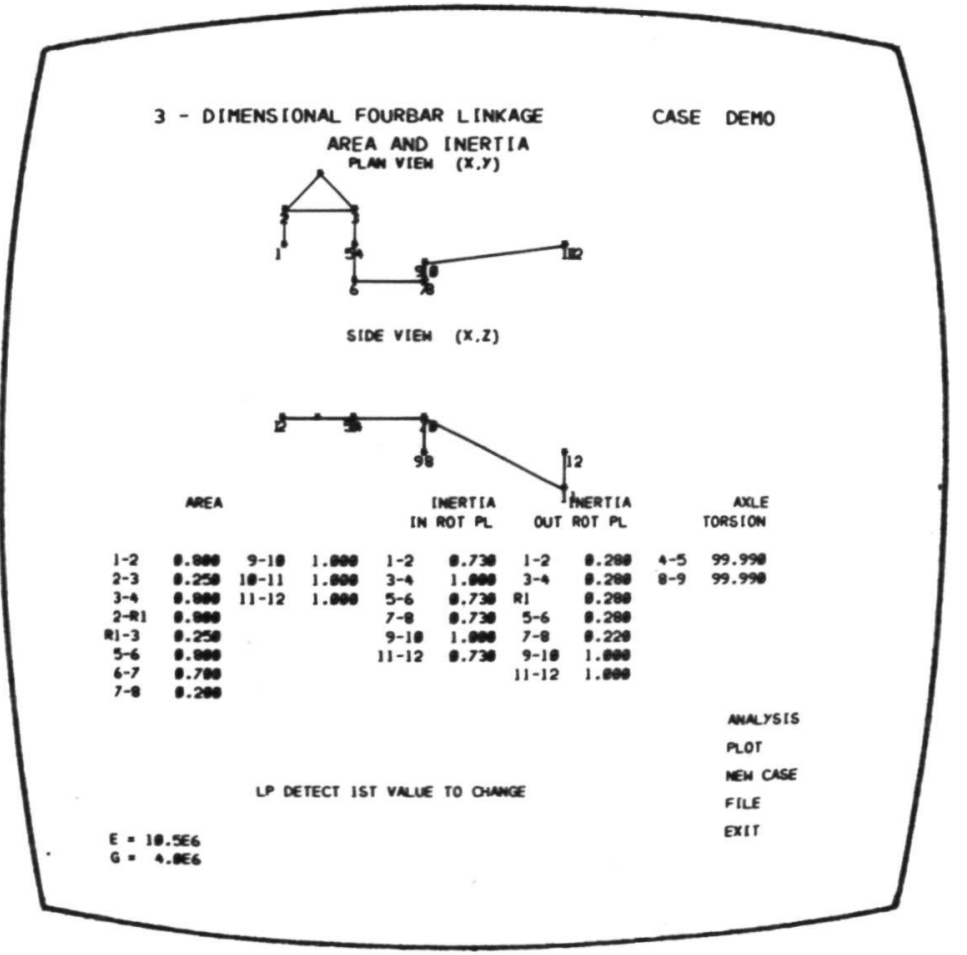

FIgure 11 - Area and Inertia Menu - displays the stiffness parameters of the given mechanism. 


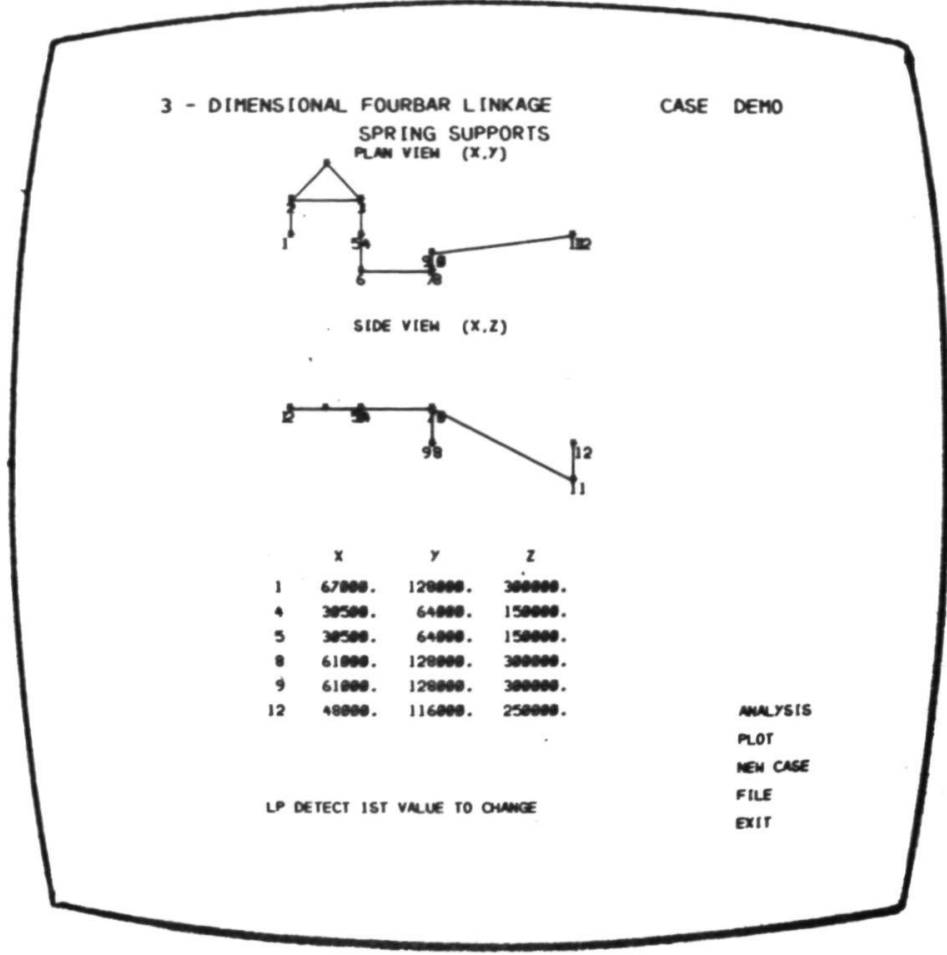

Figure 12 - Spring Support Menu - shows the X, Y, and Z spring supports on the fixed position nodes of the mechanism.

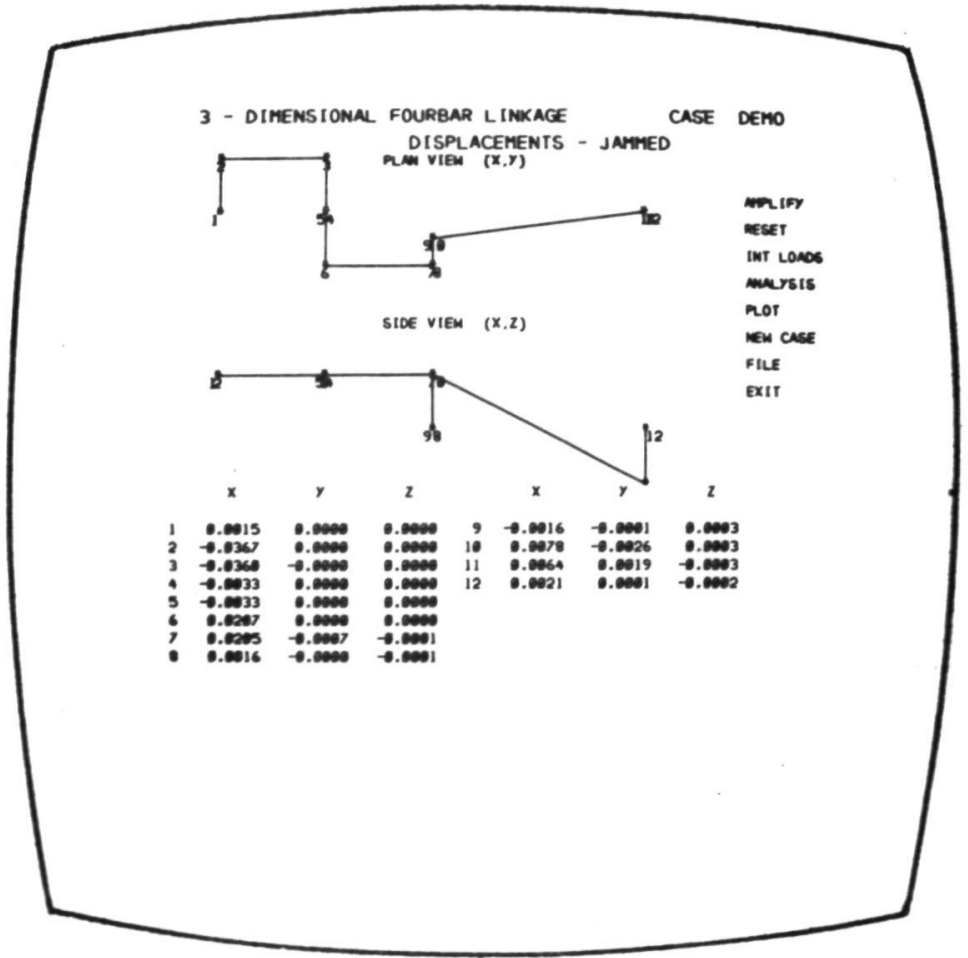

Figure 13 - D1splacement D1splay showing internal displacements of each node point in model. 


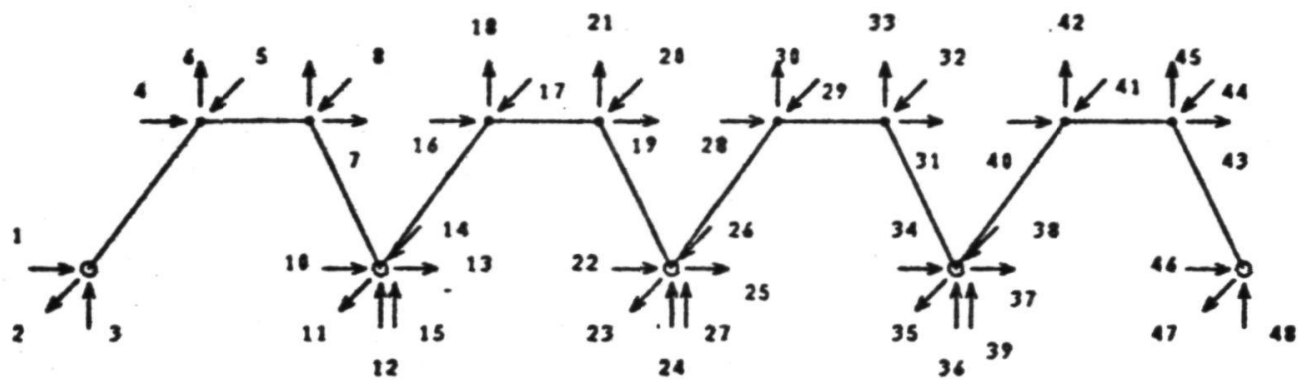

SYSTEM NODES

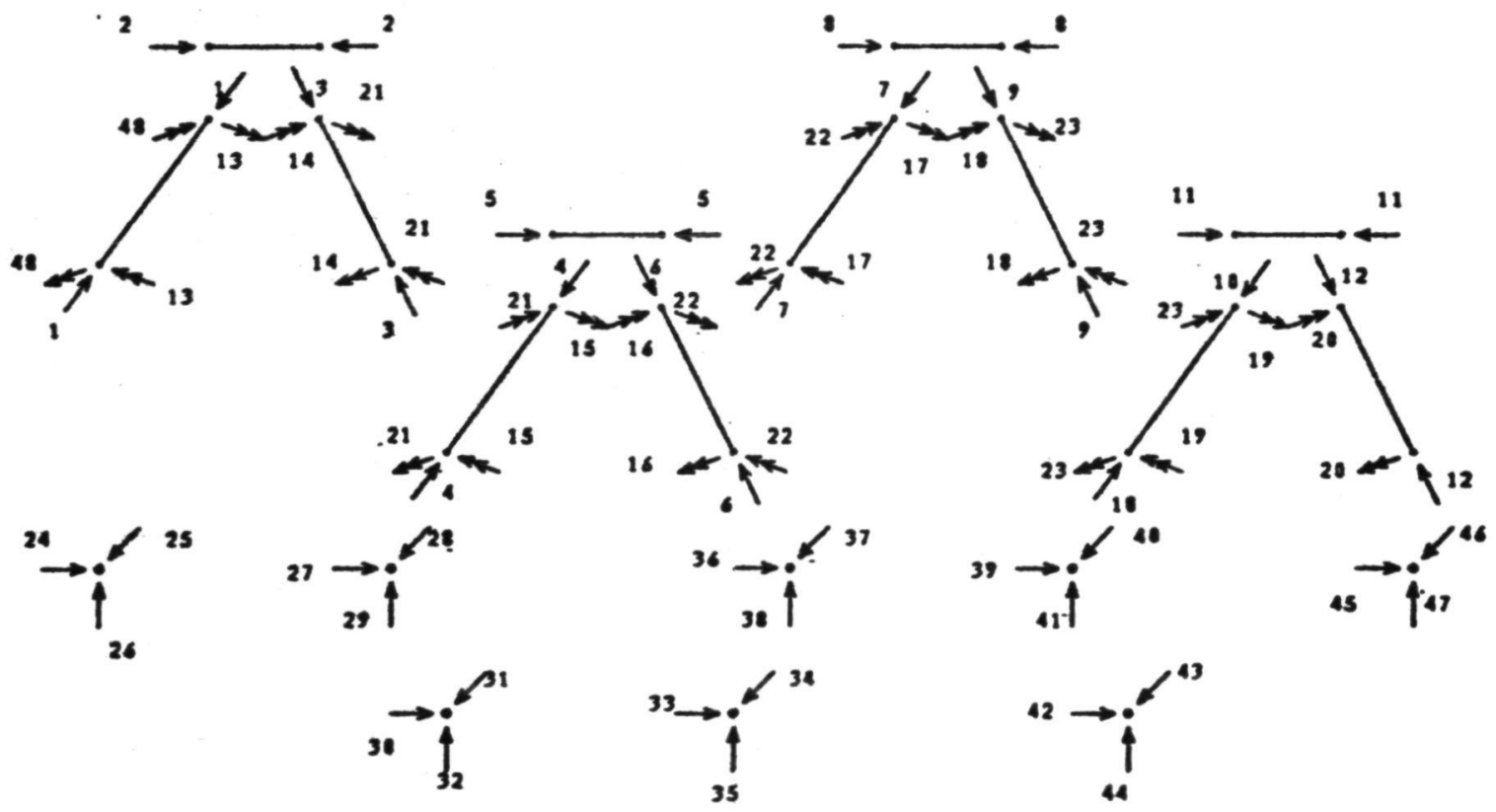

ELEMENT NODES

Figure 14 - Stick model detailing finite element system and element node relationship. 